03

\title{
Влияние процесса осадкообразования на фильтрацию жидкости из суспензии
}

\author{
(C) T.P. Аманбаев
}

Южно-Казахстанский государственный университет им. М. Ауэзова, 160012 Шымкент, Казахстан

e-mail: tulegen_amanbaev@mail.ru

(Поступило в Редакцию 8 августа 2016 г.)

Рассмотрена задача о гравитационной фильтрации жидкости в суспензии с учетом процесса формирования осадка седиментирующими дисперсными частицами. Для описания поведения суспензии использована система уравнений одномерного безынерционного движения двухфазной смеси, а для описания движения жидкости через пористый слой осадка - уравнение фильтрации, основанное на законе Дарси. Изучена динамика формирования осадка во времени. Отдельно исследованы предельные случаи равноплотной и равновесной суспензий. Получены формулы для интегральных характеристик процесса фильтрования дисперсной смеси в гравитационном поле, и проведено сравнение интенсивностей фильтрации в разных режимах. Исследовано влияние определяющих параметров на динамику процесса фильтрации жидкости из суспензии.

DOI: 10.21883/JTF.2017.05.44438.2019

\section{Введение}

Во многих практических задачах возникает необходимость изучения процессов, происходящих при фильтрации суспензии через пористый слой (например, в вопросах очистки жидкостей от содержащихся в них примесей путем фильтрования, при изучении проблемы снижения проводимости прискважинной зоны пласта за счет проникновения в него фильтрата промывочной жидкости и др.) [1-8]. Важной особенностью этих задач является наличие многообразия сложных явлений, таких как неньютоновское реологическое состояние среды, седиментация дисперсных включений, расслоение составляющих фаз, осадкообразование, фильтрация несущей

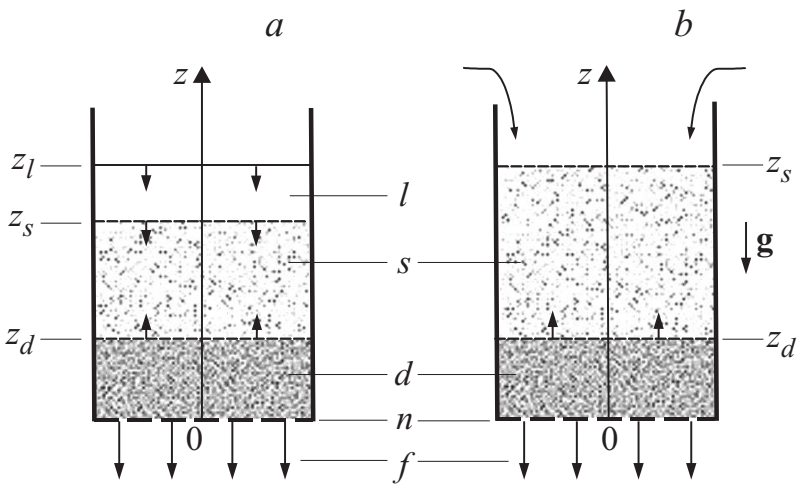

Pис. 1. Схема гравитационной фильтрации суспензии с образованием осадка в одномерной постановке: $a-$ при отсутствии притока суспензии извне (постановка $A$ ) $[16] ; b-$ при постоянной высоте столба дисперсной смеси $\left(z_{s}=\right.$ const) с непрерывным доливанием суспензии извне (постановка $B$ ); $l$ - чистая жидкость, $s-$ суспензия, $d-$ осадок, $n-$ удерживающая сетка, $f-$ фильтрат, $z_{l}, z_{s}, z_{d}-$ координаты поверхностей (верхних границ) жидкости, суспензии и осадка. фазы через пористую стенку и др. [9-15]. Моделирование и решение гидродинамической задачи с учетом вышеупомянутых явлений вызывает большие трудности. В частности, граничные условия необходимо ставить на подвижной поверхности раздела между осадком и суспензией, положение которой, вообще говоря, заранее неизвестно.

Анализ литературы показывает, что, несмотря на большое число работ, посвященных исследованию гидромеханических процессов разделения неоднородных сред, решение данной проблемы еще далеко от завершения. При этом особое значение имеет изучение влияния процесса формирования осадка на характер поведения фильтруемой двухфазной среды.

В этой связи в [16] построена математическая модель задачи гравитационного фильтрования дисперсной смеси с учетом процессов седиментации дисперсной фазы, фильтрации жидкости и осадкообразования, и исследована динамика суспензии, находящейся в изолированном сосуде в одномерной постановке, примерная схема которой показана на рис. 1, $a$. В данной работе аналогичная задача рассматривается в другой постановке, когда в сосуд по мере вытекания жидкости непрерывно подается дополнительная масса суспензии для поддержания неизменной высоты столба дисперсной смеси (рис. 1,b).

\section{1. Основные допущения и уравнения}

\section{1. Основные допущения}

Рассмотрим процесс фильтрации однородной двухфазной суспензии несжимаемой жидкости с седиментирующими твердыми сферическими частицами в поле силы тяжести (рис. 1, $b$ ). Предположим, что жидкость может просачиваться через осадок, удерживаемый снизу 
сеткой, которая не мешает жидкости вытекать на нижней границе осадка. По мере вытекания жидкости в сосуд доливается суспензия так, чтобы слой дисперсной смеси имел постоянную высоту. Под действием силы тяжести и фильтрации жидкости частицы движутся вниз, и происходит формирование осадка, высота которого со временем растет по мере осаждения частиц из суспензии. Слой осадка примем как однородную недеформируемую пористую среду, состоящую из плотной упаковки сферических монодисперсных частиц. Процесс фильтрации жидкости из суспензии через осадок с увеличивающейся высотой рассмотрим до тех пор, пока слой осадка не достигнет максимальной высоты (т.е. поверхности суспензии). Задачу исследуем аналогично [16] в одномерной постановке (влиянием стенок пренебрегаем) и в предположении, что силами инерции из-за ускорений фаз и их сжимаемостями можно пренебречь.

В отличие от данной постановки в задаче, рассмотренной в [16], подвод суспензии извне отсутствует и вследствие этого в верхней части сосуда из-за седиментации дисперсной фазы формируется слой жидкости, свободный от частиц (рис. 1,a). При этом поверхность жидкости, а также поверхность раздела между чистой жидкостью и суспензией перемещаются вниз (каждая со своей скоростью). (Более подробно решение задачи в такой постановке см. в [16].)

В дальнейшем при сравнительном анализе для удобства разные постановки задачи гравитационного фильтрования дисперсной смеси, а именно при отсутствии притока суспензии извне и его наличии, будем именовать условно как $A$ и $B$, а характерные величины, относящиеся к разным постановкам, будут снабжаться соответствующими символами.

Далее параметры жидкой и дисперсной фаз отмечены нижними индексами 1 и 2 соответственно.

\section{2. Уравнения фильтрации жидкости}

В рамках принятых допущений уравнение одномерной фильтрации несжимаемой жидкости через пористую среду можно записать в виде [17]

$$
\Delta H=\frac{\partial^{2} H}{\partial z^{2}}=0, \quad H=\frac{p}{\rho_{1}^{0} g}+z,
$$

где $H$ - пьезометрический напор, $p$ - давление, $\rho_{1}^{0}$ - истинная плотность жидкости, $z$ - вертикальная координата (направлена вверх против силы тяжести), $g$ - ускорение силы тяжести. Как отмечено в [17], уравнение (1) годится и для описания нестационарной фильтрации. При этом зависимость от времени может учитываться как через параметры, входящие в закон фильтрации, так и через граничные условия.

Скорость фильтрации жидкости $w_{f}$ определяется по формуле (закон Дарси) [17]

$$
w_{f}=-C \operatorname{grad} H \text {. }
$$

Здесь $C$ - коэффициент фильтрации (имеет размерность скорости).
Поставим следующие граничные условия: давление на поверхности осадка примем равным гидростатическому давлению, а на дне сосуда - равным нулю (поскольку добавление произвольной константы к напору не меняет скорости фильтрации). Таким образом, для напора имеем

$$
\begin{gathered}
z=0: \quad H=0, \\
z=z_{d}: \quad H=\frac{\rho g\left(z_{s}-z_{d}\right)}{\rho_{1}^{0} g}+z_{d}, \\
\rho=\rho_{1}+\rho_{2} .
\end{gathered}
$$

Здесь $z_{s}, z_{d}-$ координаты поверхностей (верхних границ) соответственно суспензии и осадка (рис. $1, b), \rho-$ плотность суспензии, $\rho_{1}, \rho_{2}-$ приведенные плотности фаз (определены ниже). В данном случае в отличие от постановки $A$ (рис. $1, a), z_{s}=$ const.

\section{3. Уравнения движения суспензии}

Для описания поведения суспензии вне пористого осадка используем систему уравнений одномерного безынерционного движения двухфазной среды с несжимаемыми фазами [18]

$$
\begin{gathered}
\frac{\partial \alpha_{1}}{\partial t}+\frac{\partial \alpha_{1} w_{1}}{\partial z}=0, \quad \frac{\partial \alpha_{2}}{\partial t}+\frac{\partial \alpha_{2} w_{2}}{\partial z}=0 \\
-\alpha_{1} \frac{\partial p}{\partial z}-K \alpha_{1} \alpha_{2}\left(w_{1}-w_{2}\right)-\rho_{1} g=0 \\
-\alpha_{2} \frac{\partial p}{\partial z}+K \alpha_{1} \alpha_{2}\left(w_{1}-w_{2}\right)-\rho_{2} g=0 \\
\rho_{1}=\rho_{1}^{0} \alpha_{1}, \quad \rho_{2}=\rho_{2}^{0} \alpha_{2}, \quad \alpha_{1}+\alpha_{2}=1 \\
K=\frac{18 \mu}{d^{2}} \psi\left(\alpha_{2}\right), \quad \rho_{1}^{0}, \rho_{2}^{0}=\text { const. }
\end{gathered}
$$

Здесь $w_{1}, w_{2}, \alpha_{1}, \alpha_{2}$ - соответственно скорости и объемные доли несущей и дисперсной фаз, $\rho_{2}^{0}-$ истинная плотность дисперсной фазы, $d-$ диаметр частиц, $\mu-$ вязкость жидкости, $\psi$ - коэффициент, учитывающий влияние объемного содержания дисперсных частиц на силу, приходящуюся на одну частицу (или коэффициент, учитывающий стесненность осаждения) и зависящий от структуры расположения частиц, $t-$ время. Уравнения (4) - это уравнения неразрывности несущей и дисперсной фаз, (5), (6) - уравнения сохранения импульсов фаз, где сила межфазного взаимодействия задана в соответствии с законом Стокса с учетом стесненности частиц, задаваемой коэффициентом $\psi[18]$. Эффект стесненности движущихся частиц в дисперсных смесях изучен достаточно подробно и для коэффициента стесненности частиц, исходя из различных схем их расположения, получены разные формулы [18-20]. В частности, схема ползущего течения вокруг хаотически расположенных частиц приводит к формуле

$$
\psi\left(\alpha_{2}\right)=\left(1-\alpha_{2}\right)^{-m}, \quad m=3-5 .
$$


Таким образом, система уравнений (1), (4)-(6) с граничными условиями (2), (3) и замыкающими соотношениями (7), (8) представляет собой модель, описывающую процесс фильтрования суспензии под действием силы тяжести с учетом эффектов осадкообразования и стесненности осаждения дисперсной фазы в одномерном приближении.

\section{2. Постановка задачи}

\section{1. Скорость перемещения поверхности осадка}

Решением уравнения (1) является линейная функция, которая с учетом граничных условий (2), (3) примет вид

$$
\begin{gathered}
H=a z, \\
a=\frac{z_{s}+\gamma\left(z_{s}-z_{d}\right)}{z_{d}}, \quad \gamma=\alpha_{2}\left(\frac{\rho_{2}^{0}}{\rho_{1}^{0}}-1\right) .
\end{gathered}
$$

При этом скорость фильтрации жидкости равна

$$
w_{f}=-C \operatorname{grad} H=-C a,
$$

a распределение давления вдоль осадка имеет форму $p=(a-1) \rho_{1}^{0} g z$.

Таким образом, в этой задаче, как и в задаче типа $A([16])$, скорость фильтрации в каждый момент времени постоянна во всех точках пористой среды и направлена вертикально вниз, а давление жидкости изменяется вдоль осадка по линейному закону. Важно отметить, что величина $H$, определяемая формулой (9), зависит не только от координаты, но и от времени (неявно), поскольку координата поверхности осадка $z_{d}$, от которой она зависит, меняется со временем.

Из уравнений (4)-(6) нетрудно определить отдельно скорости жидкой и дисперсной фаз суспензии (подробности см. в [16]). В частности, для скорости твердой фазы имеем

$$
w_{2}=w_{f}-w_{p},
$$

$w_{p}=\frac{\alpha_{1}\left(-w_{p}^{0}\right)}{\psi\left(\alpha_{2}\right)}, w_{p}^{0}=\frac{\left(\rho_{1}^{0}-\rho_{2}^{0}\right) g d^{2}}{18 \mu}\left(w_{f} \leq 0, w_{p}>0\right)$,

где $w_{p}^{0}-$ скорость дрейфа или витания одиночной частицы в жидкости (в данном случае $\left.w_{p}^{0}<0\right)$. Величина $w_{p}$ представляет собой скорость осаждения частиц при отсутствии фильтрации с учетом эффектов стесненности (отвечает коэффициент $\psi$ ) и вытеснения жидкости вверх осаждающимися частицами (отвечает множитель $\left.\alpha_{1}\right)$ [18]. Из (11) видно, что наличие фильтрации приводит к увеличению скорости осаждения частиц.

На поверхности осадка происходит скачок параметров несущей и дисперсной фаз, на котором выполняются условия Ренкина-Гюгонио. Отсюда для скорости перемещения поверхности осадка $w_{d}$ получим следующую формулу:

$$
w_{d}=-\frac{\alpha_{2}}{\alpha_{2 d}-\alpha_{2}} w_{2}
$$

Здесь $\alpha_{2 d}$ - объемная концентрация частиц в осадке $\left(\alpha_{2 d}>\alpha_{2}\right)$.
Таким образом, имеем соответствующую формулу для скорости перемещения поверхности осадка при наличии процессов седиментации частиц, фильтрации жидкости и осадкообразования.

\section{2. Уравнение движения поверхности осадка}

Для полного описания поведения суспензии при наличии вышеуказанных процессов необходимо знать закон движения поверхности осадка $z_{d}=z_{d}(t)$. При этом следует иметь в виду, что, согласно принятому выше допущению, процесс фильтрации жидкости из суспензии рассматривается до тех пор, пока поверхность осадка не достигнет максимальной высоты $z_{s}$, т.е. до выполнения условия $z_{d}=z_{s}$. Из (10) видно, что в случае $z_{d} \rightarrow z_{s}$ скорость фильтрации $w_{f}$ приближается к величине $-C$ (соответствует физическому смыслу коэффициента фильтрации [17]).

Зная скорость перемещения поверхности осадка, можно записать следующее уравнение, описывающее динамику изменения высоты осадка

$$
\frac{d z_{d}}{d t}=w_{d}\left(z_{d}\right)
$$

которое, учитывая выражения (10)-(12), можно привести к виду

$$
\begin{aligned}
& \frac{d z_{d}}{d t}=R\left[C \frac{z_{s}+\gamma\left(z_{s}-z_{d}\right)}{z_{d}}+w_{p}\right], \\
& R=\frac{\alpha}{1-\alpha}, \quad \alpha=\frac{\alpha_{2}}{\alpha_{2 d}}, \quad z_{s}=\text { const. }
\end{aligned}
$$

Примем следующее начальное условие: при $t=0$ имеем $z_{d}=z_{d 0}$. Так что в рамках принятых допущений величина $z_{d}$ меняется в интервале $z_{d 0} \leq z_{d} \leq z_{s}$. Отметим, что при $z_{d 0} \rightarrow 0$ в начальный момент времени скорость фильтрации $w_{f}$ стремится к бесконечности (свойство, характерное для задач фильтрации [17]). Однако, как установлено ниже, эта особенность в окончательном решении поставленной задачи не проявляется (т.е. при $z_{d 0} \rightarrow 0$ решение будет сходящимся). С математической точки зрения ненулевое значение $z_{d 0}$ следует рассматривать как возмущение начальных условий.

Прежде чем искать решение задачи в общем виде, целесообразно проанализировать некоторые частные случаи. При этом для удобства уравнение (13) запишем в безразмерной форме

$$
\begin{gathered}
\frac{d \bar{z}}{d \bar{t}}=R\left[\frac{\bar{\rho}}{\bar{z}}+(\bar{w}-\bar{\rho})\right], \\
\bar{\rho}=1+\gamma, \quad \bar{w}=1+\bar{w}_{p}, \quad \gamma=\alpha_{2}\left(\bar{\rho}_{2}^{0}-1\right), \\
\bar{t}=\frac{C}{z_{s}} t, \quad \bar{z}=\frac{z_{d}}{z_{s}}, \quad \bar{w}_{p}=\frac{w_{p}}{C}, \\
\bar{\rho}=\frac{\rho}{\rho_{1}^{0}}, \quad \bar{\rho}_{2}^{0}=\frac{\rho_{2}^{0}}{\rho_{1}^{0}} \quad(C \neq 0) .
\end{gathered}
$$


Уравнение (14) представляет собой уравнение Абеля второго рода (простейшего вида) [21].

Начальное условие соответственно примет вид $\bar{t}=0$, $\bar{z}=\bar{z}_{0}$. Тогда безразмерная переменная меняется от $\bar{z}_{0}$ до 1.

Далее верхнюю черту у безразмерных величин опускаем.

Для дальнейших рассуждений удобно ввести следующую функцию:

$$
G(\rho, w, z)=\rho+(w-\rho) z .
$$

Обозначим $G(\rho, 1, z)=\rho+(1-\rho) z \equiv \Gamma(\rho, z)$. Очевидно, справедливы соотношения

$$
\begin{gathered}
G(1,1, z)=1, G(\rho, \rho, z)=G(\rho, w, 0)=\rho, \\
G(\rho, w, 1)=w, \Gamma(1, z)=\Gamma(\rho, 1)=1, \Gamma(\rho, 0)=\rho,
\end{gathered}
$$

которые полезно иметь в виду при обсуждении получаемых ниже формул.

\section{3. Анализ частных случаев}

\section{1. Случай $\alpha_{2}=0$}

При $\alpha_{2}=0$ (т.е. отсутствие частиц в суспензии) параметр $R=0$, и из (13) с учетом начального условия имеем тривиальное решение $z_{d}=z_{d 0}$, что соответствует задаче об истечении жидкости из сосуда через пористый слой заданной толщины $z_{d 0}$ при постоянной высоте столба жидкости $z_{s}$ (известный опыт Дарси [17]). Скорость фильтрации в этом случае равна $w_{f}=-C z_{s} / z_{d 0}$ (совпадает с известным выражением [17]).

\section{2. Случай $d=0$}

Интересно рассмотреть другой предельный случай, когда размеры частиц достаточно малы, чтобы пренебречь их седиментацией. В этом случае $w_{p}=0$, а параметр $\gamma$ будем считать не равным нулю $\gamma \neq 0$. Тогда $w=1$, а $\rho \neq 1$. Для краткости рассматриваемый предельный случай будем обозначать по аналогии с [9], как $d=0$ („равновесная“ суспензия). В данном случае решение уравнения (14) можно выразить в неявной форме

$$
t=-\frac{1}{\gamma R}\left\{\left(z-z_{0}\right)+\frac{\rho}{\gamma} \ln \left[\frac{\Gamma(\rho, z)}{\Gamma\left(\rho, z_{0}\right)}\right]\right\}, \quad \rho=1+\gamma .
$$

При этом характерное время $t_{B}$ (здесь и далее нижние символы $A$ и $B$ указывают на тип постановки задачи), в течение которого осадок достигает максимальной высоты $z=1\left(z_{d}=z_{s}\right)$, вычисляется по формуле

$$
t_{B}=-\frac{1}{\gamma R}\left[\left(1-z_{0}\right)-\frac{\rho}{\gamma} \ln \Gamma\left(\rho, z_{0}\right)\right] \text {. }
$$

$\mathrm{B}$ частности, при $z_{0}=0$ с учетом (16) имеем

$$
t_{B}=-\frac{1}{\gamma R}\left(1-\frac{\rho}{\gamma} \ln \rho\right) .
$$

В этом случае при $\rho_{2}^{0} \sim 1$ (т.е. $\gamma \ll 1$ ) для характерного времени справедливо следующее асимптотическое представление

$$
t_{B}=\frac{1}{6 R}\left[3-\gamma+O\left(\gamma^{2}\right)\right],
$$

а при $\alpha_{2} \ll 1$ и не очень большом $\rho_{2}^{0}(\lesssim 10)$ имеет место приближенное соотношение $t_{B} \cong 0.5 \alpha^{-1}$.

Отметим, что рассматриваемая схематизация $d=0$ имеет важное прикладное значение, поскольку суспензии с малыми (броуновскими) частицами часто встречаются в приложениях (наножидкости, коллоидные растворы и т.п.). Характерное время фильтрации в этом случае, как видно из (18), зависит от относительной плотности суспензии $\rho$ (или параметра $\gamma$ ) и безразмерной высоты начального слоя осадка $z_{0}$.

\section{3. Случай $\rho=w$}

При выполнении условия

$$
\rho=w \quad\left(\text { или } \gamma=w_{p}\right)
$$

правая часть уравнения (14) несколько упрощается и его решение, а также соответствующее характерное время, запишутся в виде

$$
z=\sqrt{2 R \rho t+z_{0}^{2}}, \quad t_{B}=\frac{1-z_{0}^{2}}{2 R \rho} .
$$

В частном случае „равноплотной“ (термин позаимствован из [15]) суспензии, когда $\rho_{2}^{0}=1$ (значит $\rho=w=1$ или $\left.\gamma=w_{p}=0\right)$ из (21) получим

$$
z=\sqrt{2 R t+z_{0}^{2}}, \quad t_{B}=\frac{1-z_{0}^{2}}{2 R}
$$

Анализ показал, что характерное время $t_{B}$, отвечающее равноплотной смеси и вычисляемое по (22), является наибольшим среди рассмотренных выше частных случаев. Заметим, что закон изменения высоты осадка в случае выполнения условия (20) не зависит от диаметра частиц.

Условие (20) нетрудно привести к соотношению между $\alpha_{2}$ и параметром $\kappa=g d^{2} \rho_{1}^{0} / 18 \mu C$

$$
\frac{\alpha_{2} \psi\left(\alpha_{2}\right)}{1-\alpha_{2}}=\kappa
$$

где функция $\psi\left(\alpha_{2}\right)$, отвечающая за эффект стесненности осаждения частиц, определена согласно (8). Поскольку величина $\alpha_{2}$ не превышает предельного значения $\alpha_{2}=\alpha_{2 d}$, очевидно, что существует максимальное значение параметра $\kappa$, при превышении которого соотношение (23) перестает выполняться. В частности, при значении показателя стесненности $m=5$ в выражении (8) 


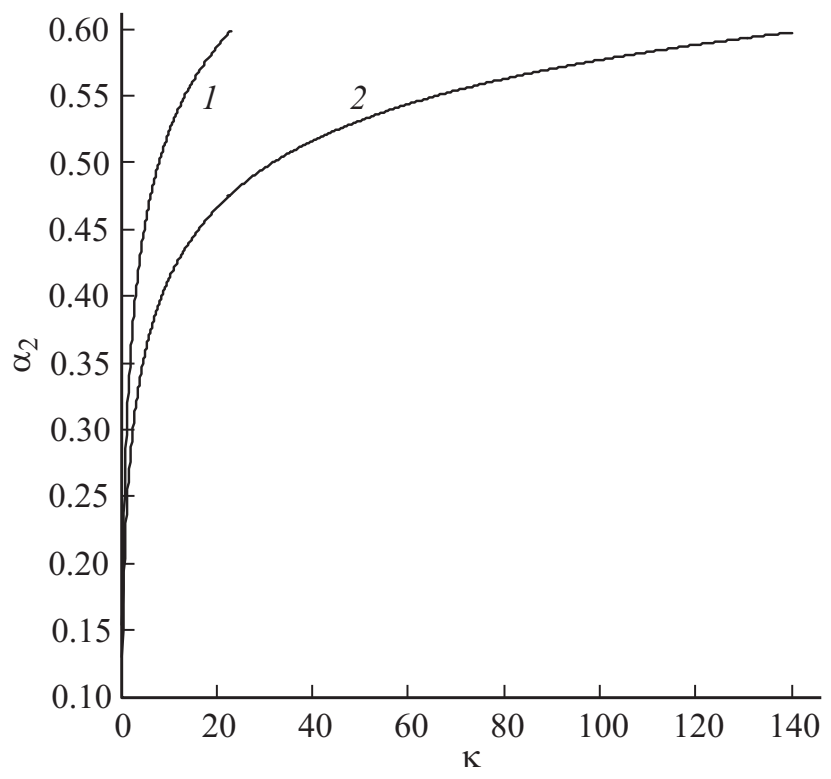

Рис. 2. Решение уравнения (23): $1-m=3 ; 2-5$;

такое максимальное значение примерно равно $\kappa \cong 146$, а в случае $m=3$ для него имеем $\kappa \cong 23$. Решение трансцендентного уравнения (23) в виде зависимости $\alpha_{2}$ от $\kappa$ при двух значениях $m=3,5$ представлено на рис. 2. Видно, что с увеличением параметра $\kappa$ значение $\alpha_{2}$, отвечающее соотношению (23), возрастает.

Необходимо подчеркнуть, что хотя частицы в предельных случаях равноплотной $\left(\rho_{2}^{0}=1\right)$ и равновесной $(d=0)$ суспензий сами по себе не подвержены седиментации, однако по мере фильтрации жидкости через нижнюю границу они накапливаются в осадке, и приводят к увеличению его высоты (следовательно, уменьшению скорости фильтрации жидкости).

\section{4. Решение задачи в общем случае}

Решение уравнения (14) с принятым начальным условием в общем случае имеет неявный вид

$$
t=\frac{1}{(w-\rho) R}\left\{\left(z-z_{0}\right)-\frac{\rho}{w-\rho} \ln \left[\frac{G(\rho, w, z)}{G\left(\rho, w, z_{0}\right)}\right]\right\},
$$

где функция $G$ определена согласно (15). Характерное время, соответствующее решению (24), равно

$$
t_{B}=\frac{1}{(w-\rho) R}\left\{\left(1-z_{0}\right)-\frac{\rho}{w-\rho} \ln \left[\frac{w}{G\left(\rho, w, z_{0}\right)}\right]\right\} .
$$

В частном случае $z_{0}=0$ из (24) и (25) с учетом (16) следует

$$
\begin{gathered}
t=\frac{1}{(w-\rho) R}\left\{z-\frac{\rho}{w-\rho} \ln \left[\frac{G(\rho, w, z)}{\rho}\right]\right\}, \\
t_{B}=\frac{1}{(w-\rho) R}\left[1-\frac{\rho}{w-\rho} \ln \left(\frac{w}{\rho}\right)\right] .
\end{gathered}
$$

Важно отметить, что рассмотренное выше решение, соответствующее частному случаю $\rho=w$, непосредственно не вытекает из решения задачи для общего случая (24), так как при $\rho=w$ в правой части (24) возникает неопределенность типа $\infty \cdot 0$. В этом смысле обсуждаемые ранее решения (21) и вытекающее из него специальное решение (22) можно трактовать как „особые“, а условие (20) - условием бифуркации решения уравнения (14). Что касается зависимости (17), соответствующей равновесной суспензии, то она является пределом общего случая (24) при уменьшении диаметра частиц $d \rightarrow 0$.

Когда $\rho \sim w$ (или $\left.\gamma \sim w_{p}\right)$, разлагая логарифмическую функцию в ряд по малому параметру и оставляя в нем первые три слагаемые, для выражения (24) получим следующее асимптотическое представление:

$$
\begin{gathered}
t=\frac{1}{6 \rho R}\left[3\left(z^{2}-z_{0}^{2}\right)-2 \varepsilon\left(z^{3}-z_{0}^{3}\right)+O\left(\varepsilon^{2}\right)\right], \\
\varepsilon=\frac{w-\rho}{\rho}, \quad|\varepsilon| \ll 1,
\end{gathered}
$$

которое при $\varepsilon \rightarrow 0$ переходит в соответствующее решение (21). Таким образом, установленное приближенное соотношение показывает, что имеет место асимптотический переход между решениями поставленной задачи в общем и частном случаях при критической ситуации $\rho \rightarrow w$.

Отметим, что особенность поведения скорости фильтрации при $z_{d 0} \rightarrow 0$ в начальный момент времени, о которой говорилось выше, в решениях задачи никак не проявляется, о чем можно убедиться непосредственной проверкой.

\section{5. Интегральные характеристики фильтрования суспензии}

\section{1. Удельный объем жидкости}

Вычислим объем жидкости $V_{B}$, вытекший из сосуда за характерное время $t_{B}$ через единицу площади поперечного сечения сосуда (удельный объем с размерностью длины)

$$
V_{B}=\int_{0}^{t_{B}}\left|w_{f}\right| d t=\int_{0}^{t_{B}} C a d t=\int_{0}^{t_{B}} C \frac{z_{s}+\gamma\left(z_{s}-z_{d}\right)}{z_{d}} d t .
$$

Переходя с помощью (13) от $d t$ к $d z_{d}$, а затем к безразмерным переменным (удельный объем $V_{B}$ отнесем к $z_{s}$, сохраняя при этом прежнее обозначение), из (26) получим интеграл, вычисляя который придем к формуле

$$
\begin{gathered}
V_{B}=\frac{1}{R} \int_{z_{0}}^{1} \frac{\Gamma(\rho, z)}{G(\rho, w, z)} d z=-\frac{1}{(w-\rho) R} \\
\times\left\{\gamma\left(1-z_{0}\right)-\frac{w_{p} \rho}{w-\rho} \ln \left[\frac{w}{G\left(\rho, w, z_{0}\right)}\right]\right\}, \\
w=1+w_{p} .
\end{gathered}
$$


В предельном случае равновесной суспензии $d=0$ из (27) для $V_{B}$ получим достаточно простое соотношение

$$
V_{B}=\frac{1-z_{0}}{R} .
$$

При выполнении условия $\rho=w$ формула (27), как и вышеприведенные формулы, приводит к неопределенности. Чтобы избежать этого, переход от $d t$ к $d z_{d}$ в (26) осуществляем с помощью уравнения, вытекающего из (13) в соответствии с рассматриваемым условием. Тогда имеем

$$
V_{B}=\frac{1-z_{0}}{2 \rho R}\left[1+\Gamma\left(\rho, z_{0}\right)\right] .
$$

Для равноплотной суспензии, когда $\rho=1$, отсюда следует

$$
V_{B}=\frac{1-z_{0}}{R} .
$$

Интересно, что для равноплотной и равновесной суспензий величина $V_{B}$ получается одинаковой. Анализ показал, что удельный объем $V_{B}$ в этих предельных случаях принимает свое наибольшее значение.

Заметим, что, хотя интеграл (26) при $z_{d 0}=0$ оказывается несобственным (из-за равенства нулю значения $z_{d}$ при $t=0)$, тем не менее, как видно из (27), он является сходящимся.

\section{2. Интенсивность фильтрации}

Имеет смысл ввести среднюю скорость фильтрации (или средний удельный расход) жидкости за время $t_{B}$, характеризующую интенсивность вытекания жидкости из суспензии и определяемую как $W_{B}=V_{B} / t_{B}$ (величина $W$ отнесена к коэффициенту фильтрации $C$ ).

C помощью величины $W_{B}$ можно оценить интенсивности процесса фильтрования дисперсных смесей в разных режимах. Например, в случае $\rho=w$ и $z_{d 0}=0$ получим $W_{B}=1+\rho$. Отсюда для равноплотной суспензии $(\rho=1)$ имеем $W_{B}=2$, т.е. в этом случае средняя скорость фильтрации жидкости ровно в два раза больше значения коэффициента фильтрации $C$. В случае равновесной суспензии величина $W_{B}$ вычисляется по формуле

$$
W_{B}=\frac{\gamma^{2}}{\rho \ln \rho-\gamma}, \quad \rho=1+\gamma .
$$

причем для достаточно малых значений параметpa $\gamma \ll 1$ справедлива приближенная формула $W_{B}=$ $=6 /(3-\gamma)$. В частности, при $\gamma \rightarrow 0(\rho \rightarrow 1)$ получим $W_{B} \rightarrow 2$.

Отметим, что в общем случае при $\alpha_{2} \rightarrow 0$ величины $V_{B}$ и $t_{B}$ стремятся к бесконечности, однако средняя скорость фильтрации $W_{B}$, определяемая их отношением, имеет конечный предел, зависящий от параметра $w_{p}$ и равный

$$
W_{B}=\frac{w_{p} \ln w}{w_{p}-\ln w}, \quad w=1+w_{p} .
$$

Аналогично в другом крайнем случае, когда $\alpha_{2} \rightarrow \alpha_{2 d}$, характерные величины $V_{B}$ и $t_{B}$ устремляются к нулю, но средняя скорость фильтрации при этом оказывается конечной и определяется по формуле

$$
W_{B}=-\frac{\gamma_{d}+w_{d} Q}{1+Q}, \quad Q=\frac{\rho_{d}}{w_{d}-\rho_{d}} \ln \left(\frac{\rho_{d}}{w_{d}}\right),
$$

где параметры $\gamma_{d}, \rho_{d}, w_{d}$ вычисляются по формулам для $\gamma, \rho, w$ при $\alpha_{2}=\alpha_{2 d}$.

Для сравнения интенсивностей фильтрования суспензии в разных постановках $A$ и $B$ (см. выше) введем параметр $\eta=W_{B} / W_{A}$, где $W_{A}-$ интенсивность (или средняя скорость) вытекания жидкости из суспензии в случае постановки $A$, т.е. при отсутствии притока суспензии извне. Приведем формулы, необходимые для расчета величины $W_{A}=V_{A} / t_{A}$ в частном случае отсутствия первоначального слоя осадка $z_{d 0}=0$. При этом воспользуемся результатами [16]. Удельный объем жидкости, вытекший из суспензии за время фильтрования $t_{A}$ (устанавливаемого выполнением условия $z_{l}=z_{d}$ (рис. $1, a$ ) определяется по формуле $V_{A}=1-\alpha$. Сопоставляя эту формулу с формулой (27), легко заметить, что по сравнению с постановкой $B$ в постановке типа $A$ удельный объем вытекшей жидкости выражается более компактной формулой.

Для характерного времени $t_{A}$ в случаях равновесной и равноплотной суспензий соответственно имеем [16]

$$
\begin{gathered}
t_{A}=\frac{\alpha(1-\alpha)}{\alpha-\rho}\left[1+\frac{\rho}{\alpha-\rho} \ln \left(\frac{\rho}{\alpha}\right)\right], \\
t_{A}=-\alpha\left(1+\frac{1}{1-\alpha} \ln \alpha\right) .
\end{gathered}
$$

Так что в рассматриваемых предельных ситуациях для интенсивности фильтрации в рамках постановки $A$ получим следующие выражения:

$$
\begin{gathered}
W_{A}=\frac{(\alpha-\rho)^{2}}{\alpha[(\alpha-\rho)+\rho \ln (\rho / \alpha)]}, \\
W_{A}=\frac{(\alpha-1)^{2}}{\alpha[(\alpha-1)-\ln \alpha]} .
\end{gathered}
$$

В общем случае величина $t_{A}$ определяется по формуле [16]

$$
t_{A}=t_{*}+\alpha \ln \left(1+\frac{w_{p} t_{*}}{\alpha}\right),
$$

где $t_{*}-$ безразмерное время полного осаждения частиц, вычисляемое из следующего неявного уравнения:

$$
\begin{gathered}
F\left(\lambda_{1}, t_{*}\right)=F\left(\lambda_{2}, t_{*}\right), \\
F\left(\lambda, t_{*}\right)=\left[\frac{\left(\lambda-w_{p}\right) t_{*}-\alpha+A(\lambda)}{-1+A(\lambda)}\right]^{\lambda-w_{p}}, \\
A(\lambda)=\left[\frac{\lambda+\gamma\left(\lambda-w_{p}\right)}{w_{p}}\right] .
\end{gathered}
$$


Здесь $\lambda_{1}, \lambda_{2}-$ корни квадратного уравнения

$$
\begin{gathered}
\lambda^{2}-b \lambda+c=0, \\
b=w_{p}+\frac{\rho-\alpha}{\alpha}, \quad c=\gamma_{d} w_{p} .
\end{gathered}
$$

В [16] установлено, что корни данного уравнения действительны и различны. Уравнение (31) представляет собой трансцендентное уравнение, для решения которого необходимо привлечь численные методы.

Нетрудно убедиться, что как для равноплотной, так и равновесной суспензий, в крайних ситуациях $\alpha_{2} \rightarrow 0$ и $\alpha_{2} \rightarrow \alpha_{2 d}$ параметр $\eta$ принимает соответственно предельные значения 0 и 1.

\section{6. Результаты расчетов}

Далее обсудим некоторые результаты расчетов, представленные на рис. 3-6. В качестве примера рассматривалась фильтрация двухфазной смеси воды с частицами кварцевого песка при нормальных условиях (соответствующие значения параметров, характеризующих физические свойства фаз, взяты из [22]). При этом значение коэффициента фильтрации принималось равным $C=10^{-3} \mathrm{~m} / \mathrm{s}$ (типичные значения $C$ при фильтрации воды в песке имеют порядок $\left.10^{-5}-10^{-2} \mathrm{~m} / \mathrm{s}[17]\right)$. Объемная концентрация твердой фазы в осадке $\alpha_{2 d}=0.6$ (значение, соответствующее, согласно опытным данным, случайной упаковке сферических частиц $[23,24])$. Считалось, что начальный слой осадка в емкости отсутствует $z_{d 0}=0$.

Для сравнения на рис. 3-6 пунктирной и штрихпунктирной кривыми показаны соответствующие зависимости в случаях равновесной $(d=0)$ и равноплотной $\left(\rho_{2}^{0}=1\right)$ суспензий, а штриховой кривой в специальном случае $\rho=w$. Так что в точках пересечения штриховой кривой со сплошными выполняется особое условие (20), причем значения анализируемых величин вдоль сплошных кривых в этих точках достигаются в пределе, поскольку используемые для построения этих кривых формулы при выполнении данного условия, как отмечено выше, приводят к неопределенности.

Очевидно, пунктирная и штрихпунктирная кривые, отвечающие равновесной и равноплотной суспензиям, представляют собой крайние положения кривых анализируемых зависимостей при уменьшении соответственно размера и истинной плотности дисперсных частиц.

На рис. 3 представлена зависимость координаты поверхности осадка $z$ (нижние кривые) и скорости фильтрации $\left|w_{f}\right| / C$ (верхние кривые) от времени $t$ при объемном содержании дисперсной фазы в смеси $\alpha_{2}=0.1$.

Видно, что с увеличением размера частиц процесс осадкообразования ускоряется, при этом скорость фильтрации замедляется. С уменьшением диаметра частиц кривая зависимости $z(t)$ приближается к пунктирной

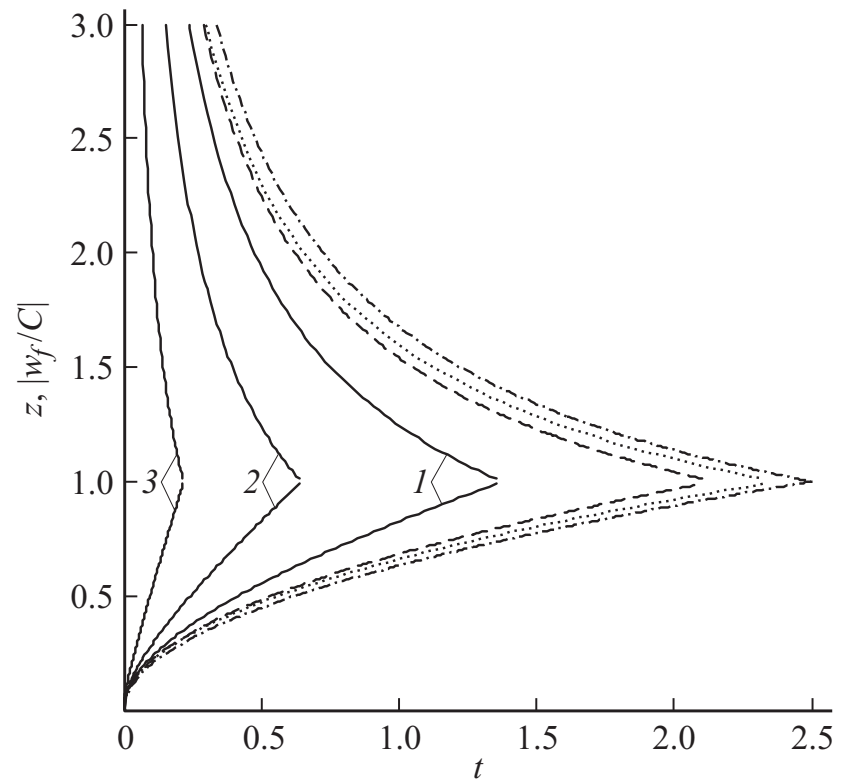

Рис. 3. Изменение координаты поверхности осадка во времени при $\alpha_{2}=0.1: 1-d=50,2-100,3-200 \mu \mathrm{m}$. Пунктирная кривая - равновесная суспензия $(d=0)$, штриховая кривая - случай $\rho=w\left(\gamma=w_{p}\right)$, штрихпунктирная кривая равноплотная суспензия $\left(\rho_{2}^{0}=1\right)$.

кривой, соответствующей равновесной суспензии. Отметим, что штрихпунктирная кривая, отвечающая равноплотной суспензии, является предельной для закона перемещения поверхности осадка при наличии процессов фильтрации жидкости, седиментации дисперсной фазы и осадкообразования, так что все кривые, описывающие динамику накопления осадка, будут располагаться выше этой кривой. Следует подчеркнуть, что для крупнодисперсных суспензий $(d \gtrsim 200 \mu \mathrm{m})$ процесс роста высоты осадка во времени приближается к линейной зависимости. Поведение скорости фильтрации и поверхности осадка в предельных ситуациях равновесной и равноплотной суспензий не сильно отличаются между собой.

На рис. 4 показана зависимость характерного времени $t_{B}$ от объемной концентрации $\alpha_{2}$ при разных диаметрах частиц дисперсной фазы. Видно, что с ростом $\alpha_{2}$ характерное время фильтрации $t_{B}$ уменьшается, стремясь к нулю с приближением $\alpha_{2}$ к предельному значению $\alpha_{2 d}$. Причем увеличение размера частиц приводит к уменьшению характерного времени $t_{B}$.

Важно отметить некоторую особенность поведения зависимости $t_{B}\left(\alpha_{2}\right)$ : после быстрого падения $t_{B}$ на небольшом отрезке следует достаточно широкий интервал изменения $\alpha_{2}$, где величина $t_{B}$ плавно уменьшается до нулевого значения в точке $\alpha_{2}=\alpha_{2 d}$. Например, в случае $d=200 \mu \mathrm{m}$ интервал медленного изменения $t_{B}$ простирается приблизительно от $\alpha_{2}=0.1$ до 0.4 . Расчеты показали, что асимптотическая формула (19) довольно хорошо аппроксимирует представленные на рис. 4 предельные зависимости. 


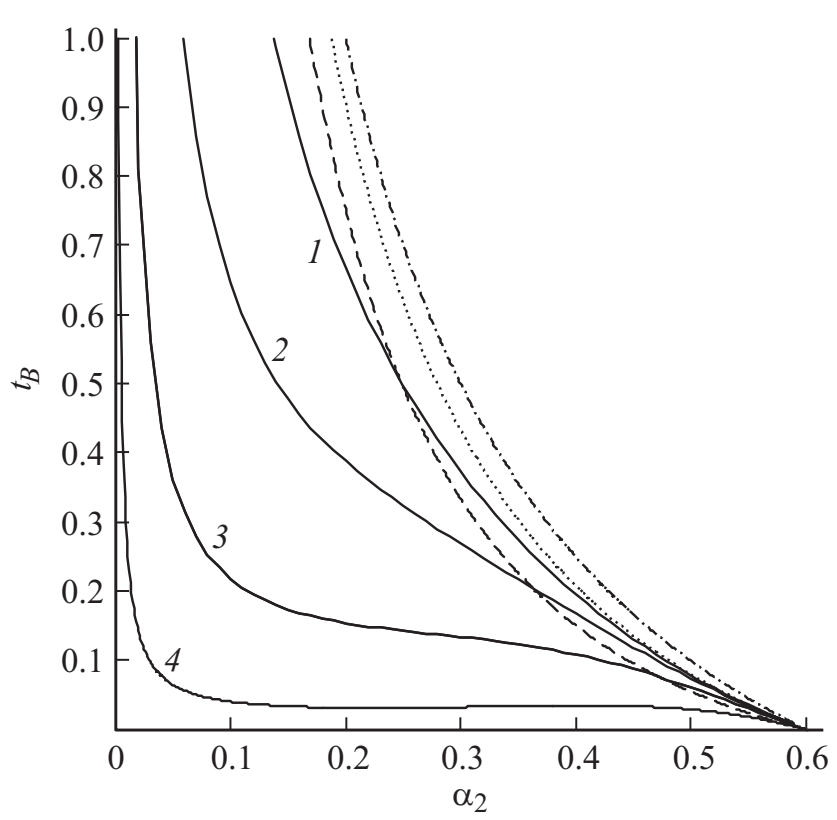

Рис. 4. Зависимости характерного времени фильтрации $t_{B}$ от $\alpha_{2}: 1-d=50,2-100,3-200,4-500 \mu \mathrm{m}$. Остальные кривые отвечают тем же случаям, что на рис. 3 .

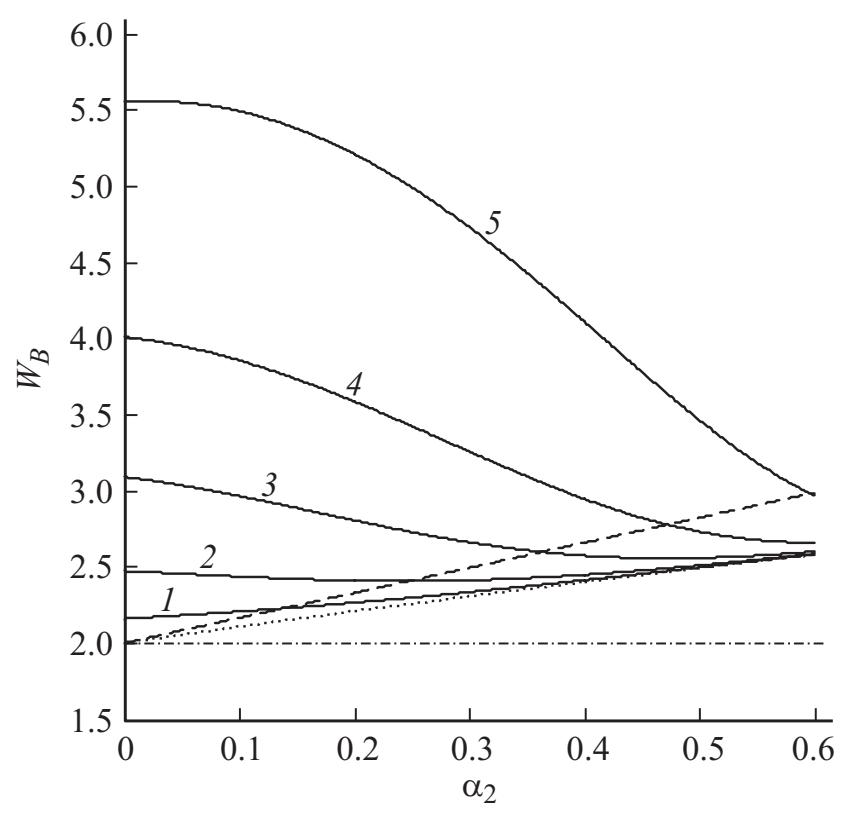

Рис. 5. Зависимости средней скорости фильтрации $W_{B}$ от $\alpha_{2}$ : $1-d=25,2-50,3-100,4-200,5-500 \mu \mathrm{m}$. Остальные кривые отвечают тем же случаям, что на рис. 3 .

Рис. 5 демонстрирует зависимость средней скорости (или интенсивности) фильтрации $W_{B}$ от объемного содержания дисперсной фазы в суспензии $\alpha_{2}$ при разных диаметрах частиц. Заметим, что когда величина $\alpha_{2}$ приближается к крайним значениям 0 и $\alpha_{2 d}=0.6$, значения $W_{B}$ устремляются к пределам, устанавливаемым формулами (29), (30) соответственно. Из представленного рисунка видно, что зависимость $W_{B}\left(\alpha_{2}\right)$ с ростом $\alpha_{2}$ может как монотонно уменьшаться (кривые 4,5), так и увеличиваться (кривая 1). Хотя она может вести себя и немонотонно, как, например, в случаях $d=50$ и $100 \mu \mathrm{m}$ (кривые 2,3 ): сначала уменьшаться, затем увеличиваться. Отметим, что характерное значение $W_{B}=2$ является наименьшим значением интенсивности фильтрации жидкости через пористый слой осадка в рассматриваемой здесь постановке.

Интересно изучить поведение параметра $\eta$, определяемого отношением интенсивностей фильтрации при наличии (постановка $B$ ) и отсутствии (постановка $A$ ) притока суспензии извне $\eta=W_{B} / W_{A}$, которое проиллюстрировано на рис. 6. Трансцендентное уравнение (31) решалось численным методом бисекции [25]. Значения $\eta$ при приближении $\alpha_{2}$ к крайним значениям 0 и $\alpha_{2 d}=0.6$ аналогично рис. 5 достигаются в пределе.

Видно, что для относительно крупных частиц $d \gtrsim 100 \mu \mathrm{m}$ зависимость $\eta\left(\alpha_{2}\right)$ ведет себя немонотонно: с ростом $\alpha_{2}$ она сначала возрастает, затем уменьшается. При этом существует некоторое критическое значение объемной концентрации, такое, что, когда $\alpha_{2}$ меньше этого значения, интенсивность фильтрации в постановке $B$ становится меньше, чем в случае постановки $A$, т.е. $\eta<1$ или $W_{B}<W_{A}$. В случае, когда объемная доля дисперсной фазы больше этого критического значения, имеет место обратное соотношение $W_{B}>W_{A}$, означающее, что средняя скорость (или интенсивность) фильтрации в постановке $B$, наоборот, больше, чем в постановке $A$. Причем значение такой критической концентрации зависит от размера частиц, например, для диаметров частиц $d=100$ и $200 \mu \mathrm{m}$ оно примерно равно 0.177 и 0.075 соответственно.

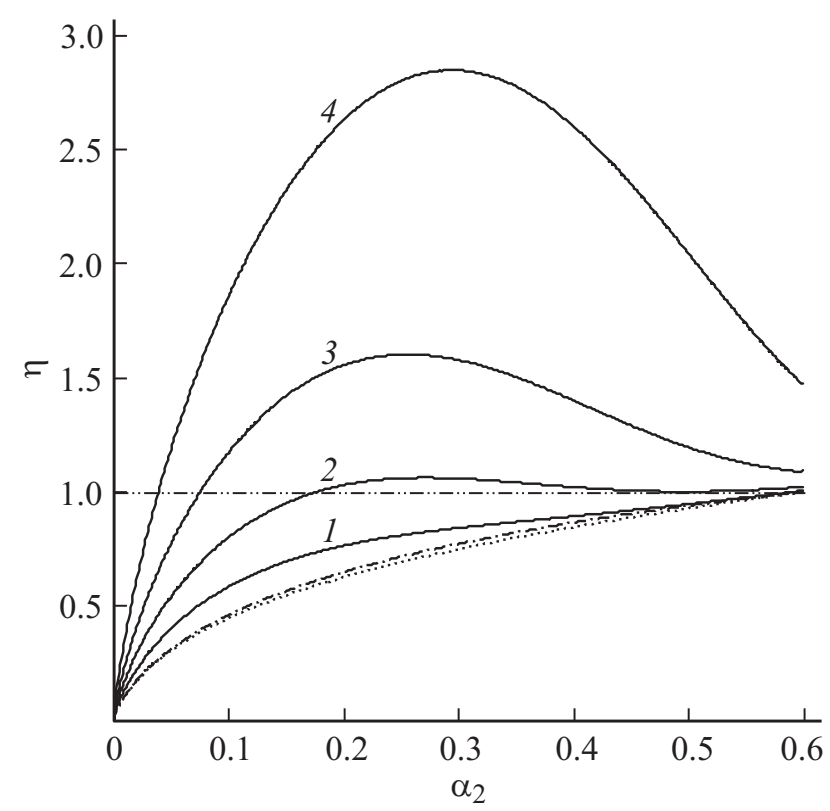

Рис. 6. Зависимости $\eta$ от $\alpha_{2}: 1-d=50,2-100,3-$ $200,4-500 \mu \mathrm{m}$. Штрихдвойнаяпунктирная линия - граница областей выполнения соотношений $\eta<1$ и $\eta>1$. Остальные кривые отвечают тем же случаям, что на рис. 3. 
Для более мелких частиц $(d \lesssim 50 \mu \mathrm{m})$, включая предельный равновесный случай $d=0$, параметр $\eta$ при изменении $\alpha_{2}$ от 0 до крайнего значения $\alpha_{2 d}$ монотонно возрастает, оставаясь при этом меньше единицы. Такое поведение величины $\eta$, как видно, характерно и для предельной равноплотной суспензии. Причем в этих предельных случаях значения $\eta$ отличаются между собой незначительно.

Заметим, что точки пересечения штриховой кривой со сплошными на вышеприведенных рисунках представляют собой точки бифуркации, поскольку в них имеет место ветвление кривой соответствующей зависимости.

\section{Заключение}

Таким образом, построена математическая модель поведения суспензии при наличии процессов фильтрации жидкости, седиментации дисперсной фазы и осадкообразования в поле силы тяжести с постоянной высотой столба дисперсной смеси (обеспечиваемой непрерывным доливанием суспензии в сосуд). На основе построенной модели проведен анализ динамики гравитационного фильтрования суспензии в широком диапазоне изменения определяющих параметров.

Следует иметь в виду, что при $\alpha_{2} \gtrsim 0.5$ дисперсная структура без контактов между частицами и с силовым взаимодействием, определяемым размером частиц и вязкостью жидкой фазы, будет нарушаться [18]. Для указанных концентраций использование уравнений движения дисперсной смеси в виде (5), (6) не соответствует физике рассматриваемого процесса. Однако это обстоятельство не нарушает общих закономерностей поведения анализируемых зависимостей. Отметим, что учет эффектов контакта частиц дисперсной фазы сильно осложняет построение адекватной модели.

Работа выполнена при финансовой поддержке Министерства образования и науки Республики Казахстан (грант № 3486/ГФ4).

\section{Список литературы}

[1] Слинько М.Г. // Теорет. основы хим. технологии. 2003. T. 37. № 5. С. 451-459.

[2] Колотыркин Я.М. // Хим. промышленность. 1984. № 10. C. 3-7.

[3] Жужсков В.А. Фильтрование. Теория и практика разделения суспензий. М.: Химия, 1971. 440 с.

[4] Sahimi M., Imdakm A.O. // Phys. Rev. Lett. 1991. Vol. 66. N 9. P. $1169-1178$.

[5] Coussy O. Poromechanics. Chichester: Wiley, 2004. 315 p.

[6] Шехтман Ю.М. Фильтрация малоконцентрированных суспензий. М.: Изд-во АН СССР, 1961. 213 с.

[7] Михайлов Н.Н. Изменение физических свойств горных пород в околоскважинных зонах. М.: Недра, 1987. 256 с.

[8] Боронин С.А., Осипцов А.А., Толмачева К.И. // Изв. РАН. Мех. жидкости и газа. 2015. № 6. С. 50-62.
[9] Нигматулин Р.И. Динамика многофазных сред. Ч. 1. М.: Наука, 1987. 464 с.

[10] Ибятов Р.И. Методы расчета гидромеханических процессов при фильтровании и центрифугировании суспензий. Автореф. док. дис. Казань: Казанский гос. архит.-строит. ун-та., 2005. $340 \mathrm{c.}$

[11] Романков П.Г. // Теор. осн. хим. технол. 1972. Т. 6. № 6. C. 855-871.

[12] Ершин Ш.А. Гидроаэродинамика. Алматы: Изд-во КазНУ, 2013. $354 \mathrm{c}$.

[13] Невский Ю.А., Осипцов А.Н. // Письма в ЖТФ. 2009. Т. 35. Вып. 7. С. $98-105$.

[14] Жуков В.Г., Чесноков В.М. // Теорет. основы хим. технологии. 2013. Т. 47. № 5. С. 584-588.

[15] Буевич Ю.А., Латкин А.Н. // Изв. АН СССР. Сер. Мех. жидкости и газа. 1991. № 2. С. 124-131.

[16] Аманбаев Т.P. // Теорет. основы хим. технологии. 2016. T. 50. № 3. C. 311-324.

[17] Леонтьев Н.Е. Основы теории фильтрации. М.: Изд-во МГУ, 2009. $88 \mathrm{c.}$

[18] Нигматулин Р.И. Динамика многофазных сред. Ч. 2. М.: Наука, 1987. 365 с.

[19] Batchelor G.K. // J. Fluid Mech. 1972. Vol. 52. P. 245-268.

[20] Головин А.М., Чижков В.Е. // Прикл. мат. и мех. 1978. Т. 42. № 1. C. 105-113.

[21] Зайцев В.Ф., Полянин А.Д. Справочник по обыкновенным дифференциальным уравнениям. М.: Физматлит, 2001. $576 \mathrm{c}$.

[22] Краткий физико-технический справочник / Под ред. К.П. Яковлева. М.: Физматлит, 1960. 448 с.

[23] Нигматулин Р.И. Основы механики гетерогенных сред. М.: Наука, 1978. 336 с.

[24] Гольдштик М.A. // Прикл. мех. и техн. физ. 1972. № 6. C. $106-112$.

[25] Амосов А.А., Дубинский Ю.А., Копченова Н.В. Вычислительные методы для инженеров. М.: Высшая школа, 1994. $544 \mathrm{c}$. 